\title{
Melatonin treatment decreases c-fos expression in a headache model induced by capsaicin
}

\author{
Fabiano C. Tanuri · Eliângela de Lima · Mario F. P. Peres · Francisco R. Cabral · \\ Maria da Graça Naffah-Mazzacoratti · Esper Abrão Cavalheiro • \\ José Cipolla-Neto · Eliova Zukerman · Débora Amado
}

Received: 17 November 2008/Accepted: 7 January 2009/Published online: 27 January 2009

(C) Springer-Verlag 2009

\begin{abstract}
The aim of the present work was to analyze c-fos response within the trigeminal nucleus caudalis (TNC) of pinealectomized rats and animals that received intraperitoneal melatonin, after intracisternal infusion of capsaicin, used to induce intracranial trigeminovascular stimulation. Experimental groups consisted of animals that received vehicle solution (saline-ethanol-Tween 80, 8:1:1, diluted 1:50) only (VEI, $n=5$ ); animals that received capsaicin solution $(200 \mathrm{nM})$ only (CAP, $n=6)$; animals submitted to pinealectomy ( $\mathrm{PX}, n=5$ ); sham-operated animals ( $\mathrm{SH}, n=5)$; animals submitted to pinealectomy followed by capsaicin stimulation ( $200 \mathrm{nM})$ after 15 days (PX $+\mathrm{CAP}, n=7)$; and animals that received capsaicin solution $(200 \mathrm{nM})$ and intraperitoneal melatonin $(10 \mathrm{mg} /$ $\mathrm{kg})(\mathrm{CAP}+\mathrm{MEL}, n=5)$. Control rats, receiving vehicle
\end{abstract}

F. C. Tanuri - E. de Lima · F. R. Cabral .

E. A. Cavalheiro - D. Amado

Departamento de Neurologia/Neurocirurgia, Universidade

Federal de São Paulo, Escola Paulista de Medicina,

São Paulo, Brazil

F. C. Tanuri - M. F. P. Peres · E. A. Cavalheiro ·

E. Zukerman · D. Amado

Hospital Israelita Albert Einstein, São Paulo, Brazil

J. Cipolla-Neto

Departamento de Fisiologia e Biofísica, Instituto de Ciências

Biomédicas, Universidade de São Paulo, São Paulo, Brazil

M. da Graça Naffah-Mazzacoratti

Departamento de Bioquímica, Universidade Federal de São

Paulo, Escola Paulista de Medicina, São Paulo, Brazil

F. C. Tanuri ( $\bigotimes)$

Rua Guanás, 77 Jardim Salgado Filho,

Marília (SP) CEP: 17502-560, Brazil

e-mail: ftanuri@yahoo.com.br in the cisterna magna, showed a small number of c-fospositive cells in the TNC (layer I/II) as well as the shamoperated and pinealectomized rats, when compared to animals stimulated by capsaicin. On the other hand, pinealectomized rats, which received capsaicin, presented the highest number of c-fos-positive cells. Animals receiving capsaicin and melatonin treatment had similar expression of the vehicle group. Our data provide experimental evidence to support the role of melatonin and pineal gland in the pathophysiology of neurovascular headaches.

Keywords Headache $\cdot$ Migraine · Pinealectomy · Melatonin $\cdot \mathrm{c}$-fos $\cdot$ Experimental models

\section{Introduction}

Migraine is a common condition with significant impact in the general population [1-3]. In spite of its relevancy, migraine neurobiology has not been fully determined. Several mechanisms are important, including the trigeminovascular concept [4]. Neurovascular network is thought to be the anatomical substrate for neurovascular headaches such as migraine and cluster headaches $[5,6]$. A- $\delta$ and unmyelinated $\mathrm{C}$-fibers of the trigeminal nerve transmit painful stimuli from meninges to trigeminal nucleus caudalis (TNC) within the brain stem, via the trigeminal ganglion (TG) neurons [7]. The pathophysiology of neurovascular headaches is unclear but the involvement of trigeminovascular system is generally accepted [8].

c-fos expression within the TNC is induced experimentally after stimulation at various sites of the trigeminovascular system, such as meninges, TG and superior sagittal sinus [9]. Development of animal models and the use of direct electrical, mechanical or chemical 
irritants stimulus (capsaicin, carrageenan and blood) have been used to mimic vascular head pain [10-13].

c-fos protein is a transcription factor expressed by immediate-early response of c-fos gene and has been accepted as a marker of functional activity in neurons [14, 15]. c-fos expression in layers I and II of the TNC is used to study the activity of the sensory part of the trigeminal system [16, 17]. This expression can be attenuated by pharmacological agents used for the treatment of migraine, including sumatriptan, dihydroergotamine and valproate $[18,19]$.

Melatonin, a hormone synthesized by the pineal gland with major influence on several circadian physiological activities, is maximally produced between midnight and dawn, with low levels during the light period [20]. Melatonin has been linked to migraine and other headaches, increasing evidence supports its role in mechanisms and treatment of headache disorders [21, 22]. Some headaches have a clear seasonal and circadian pattern, such as cluster and hypnic headaches. Melatonin levels have been found decreased in both migraine and cluster headaches [23-25]. Melatonin is related to headache pathophysiology in several ways. Due to its anti-inflammatory effect, free radical scavenging process, reduction of pro-inflammatory cytokine upregulation, nitric oxide synthase activity and dopamine release inhibition, membrane stabilization, GABA and opioid analgesia potentiation, protection against glutamate neurotoxicity, neurovascular regulation, 5-HT modulation and due to its similarity with the chemical structure of indomethacin. However, little is known about the putative effect of melatonin in experimental models of headache [26].

Thus, the aim of this study is to characterize the pattern of cerebral c-fos expression after noxious trigeminal stimulation in the group that received melatonin and in pinealectomized rats, using intracisternal administration of the capsaicin to appraise the pinealectomy and the melatonin effect in head pain.

\section{Methods}

Animals

All experimental protocols were approved by the ethical committee of the Universidade Federal de São Paulo (UNIFESP) and all efforts were made to minimize animal suffering following the proposal of International Ethical Guideline for Biomedical Research [27]. Wistar adult male rats (250-300 g) housed under environmentally controlled conditions in a 12/12 h light/dark and granted free access to food and water were used. These animals were separated into four groups.
Groups

VEI $(n=5)$ : animals that received vehicle solution only; CAP $(n=6)$ : animals that received capsaicin solution $(200 \mathrm{nM})$ only; PX $(n=5)$ : animals that were submitted to pinealectomy; $\mathrm{SH}(n=5)$ : animals that were sham-operated; PX + CAP $(n=7)$ : animals submitted to pinealectomy followed by capsaicin stimulation $(200 \mathrm{nM})$ after 15 days; and CAP + MEL $(n=5)$ animals that received capsaicin solution $(200 \mathrm{nM})$ and intraperitoneal melatonin (Sigma, $10 \mathrm{mg} / \mathrm{kg}$ ) $20 \mathrm{~min}$ after capsaicin injection.

\section{Drugs}

Capsaicin solution was prepared with $3.05 \mathrm{mg}$ capsaicin (Merck) per $1 \mathrm{ml}$ of vehicle (saline-ethanol-Tween 80, 8:1:1) and diluted 1:50 (200 $\mathrm{nM})$ with saline. Vehicle was diluted 1:50 in saline.

\section{Surgical procedures}

\section{Pinealectomy}

Pinealectomy was performed following the method described by Siuciak and Dubocovich [28]. In brief, rats were anesthetized (pentobarbital, $40 \mathrm{mg} / \mathrm{kg}$ i.p.) and fixed in a stereotaxic frame. Using a pointed dental burr, a piece of bone was removed at the juncture of the lambda and the sagittal suture lines. The pineal was grasped with fine forceps and removed. The bone disk was returned to its original position and the skin flaps pulled together. In sham-operated rats, all procedures mentioned above were used, except for the fact that the pineal gland was not removed.

\section{Capsaicin stimulation}

For this procedure, all rats were anesthetized with pentobarbital $(40 \mathrm{mg} / \mathrm{kg}$ i.p.) and a surgical opening in the region between the scalp and $\mathrm{C} 1$ (first cervical vertebra) was made. An amount of $10 \mu \mathrm{l}$ of capsaicin solution (see "Drugs") was injected into the cisterna magna (over $15 \mathrm{~min}$ ) using a Hamilton syringe with the aid of a stereotaxic frame [29]. To avoid capsaicin outflow, the needle was only removed 10 min after injection.

Perfusion and immuno-histochemistry

Two hours after infusion, the rats were anesthetized with pentobarbital overdose $(120 \mathrm{mg} / \mathrm{kg})$, followed by perfusion via the ascending aorta with $0.1 \mathrm{M}$ phosphate saline buffer (PBS, $200 \mathrm{ml}, \mathrm{pH}$ 7.4) and 4\% paraformaldehyde $(200 \mathrm{ml})$ in $0.1 \mathrm{M}$ phosphate buffer (PB, pH 7.4). Brain stem with attached cervical cord was stored overnight in 
the same fixative and then placed in a cryoprotectant (30\% sucrose in $0.1 \mathrm{M} \mathrm{PB}, \mathrm{pH}$ 7.4). Coronal serial sections $(40 \mu \mathrm{m})$ were prepared on a cryostat microtome at $-20^{\circ} \mathrm{C}$ and collected in PBS with sodium azide $(0.1 \%)$ to Nissl staining and immuno-histochemistry. cfos protein expression was characterized in free-floating sections according to the following protocol. Sections were rinsed three times $5 \mathrm{~min}$ in PBS, pre-treated with $0.3 \% \mathrm{H}_{2} \mathrm{O}_{2}$ in PBS for $15 \mathrm{~min}$, rinsed three times $5 \mathrm{~min}$ in PBS and pre-incubated in $10 \%$ bovine serum albumin (Calbiochem) and 2\% normal serum (Vector) in PBS for $2 \mathrm{~h}$ at room temperature. Sections were incubated for $48 \mathrm{~h}$ at $4{ }^{\circ} \mathrm{C}$ in PBS solution containing $2 \%$ BSA, $2 \%$ normal serum, anti-c-fos antibody (1:1,000; Calbiochem) and $0.3 \%$ Triton $\mathrm{X}-100$ in PBS. Following three washes in PBS, the sections were incubated in a PBS solution contained biotinylated rabbit $\operatorname{IgG}(1: 200)$ (Vector) for $2 \mathrm{~h}$ at room temperature. Sections were rinsed three times $5 \mathrm{~min}$ in PBS and incubated with the avidinbiotin-peroxidase complex (Vector) in PBS for $1 \mathrm{~h}$ and $30 \mathrm{~min}$ at room temperature. Sections were rinsed twice $5 \mathrm{~min}$ in PBS and $5 \mathrm{~min}$ in Tris- $\mathrm{HCl}(\mathrm{pH} \mathrm{7.6)}$ and revealed with $0.06 \% 3,3^{\prime}$-diaminobenzidine tetrahydrochloride (Sigma) and with $0.002 \% \mathrm{H}_{2} \mathrm{O}_{2}$. Sections were then mounted in slides and dehydrated through alcohol to xylene and coverslipped with Entellan (Merck).

\section{Nissl staining}

Brain stem slices $(40 \mu \mathrm{m})$ were hydrated in alcohol solutions of decreased concentration followed by staining in $0.5 \%$ cresyl Violet acetate (Sigma) diluted in $0.1 \mathrm{M}$ acetate buffer $\mathrm{pH}$ 4.0. Slices were dehydrated, coverslipped and analyzed by light microscopy optic Zeiss Axiolab.

\section{Quantifications}

c-fos immunoreactive cells in the TNC layer I/II were counted at 0 to $-1 \mathrm{~mm}$ caudal to obex. Three sections per animal were analyzed. The count of c-fos immunoreactive cells was performed using magnification of $200 \times$ under a microscope optical system Zeiss Axiolab. Representative images of the brain stem slices were digitalized using NIH Image 1.61 system. Cell counting was performed blinded by three observers and the results are expressed as the mean \pm standard deviation.

\section{Statistical analysis}

Data were analyzed using one-way analyses of variance (ANOVA) followed by Tukey's $Q$ test. A value of $P<0.05$ was accepted as significant.

\section{Results}

Nissl-stained coronal sections of brain stem in control rats showed a preserved cytoarchitecture (Fig. 1a) and the TNC (layer I/II) was observable in Fig. 1b. Sections of the other groups showed similar or normal Nissl staining (data not shown).

The pattern of c-fos expression was different in the studied groups. Controls rats receiving vehicle in the cisterna magna showed a small number of c-fos-positive cells (VEI: $9.8 \pm 2.42 ; P<0.01$ ) in the TNC (layer I/II) as well as the sham-operated rats (SH: $3.26 \pm 0.15 ; P<0.001)$, pinealectomized rats (PX: $2.3 \pm 0.78 ; P<0.001$ ) and rats that received capsaicin and melatonin (CAP + MEL: $10.53 \pm 2.78 ; P<0.01)$, when compared to capsaicintreated animals (CAP: $25.7 \pm 8.78$ ). On the other hand, pinealectomized rats that received capsaicin presented the highest number of c-fos-positive cells (PX + CAP: $41.5 \pm 6.68 ; P<0.01)$. A diffuse staining in regions that were not considered in the present work was observed. The results of c-fos expression in the immuno-histochemistry and the quantification of these c-fos-positive cells are observed in Figs. 2 and 3, respectively.

\section{Discussion}

Our results show experimental evidence for the putative role of melatonin and pineal gland in the pathophysiology of neurovascular headaches. A high number of c-fos

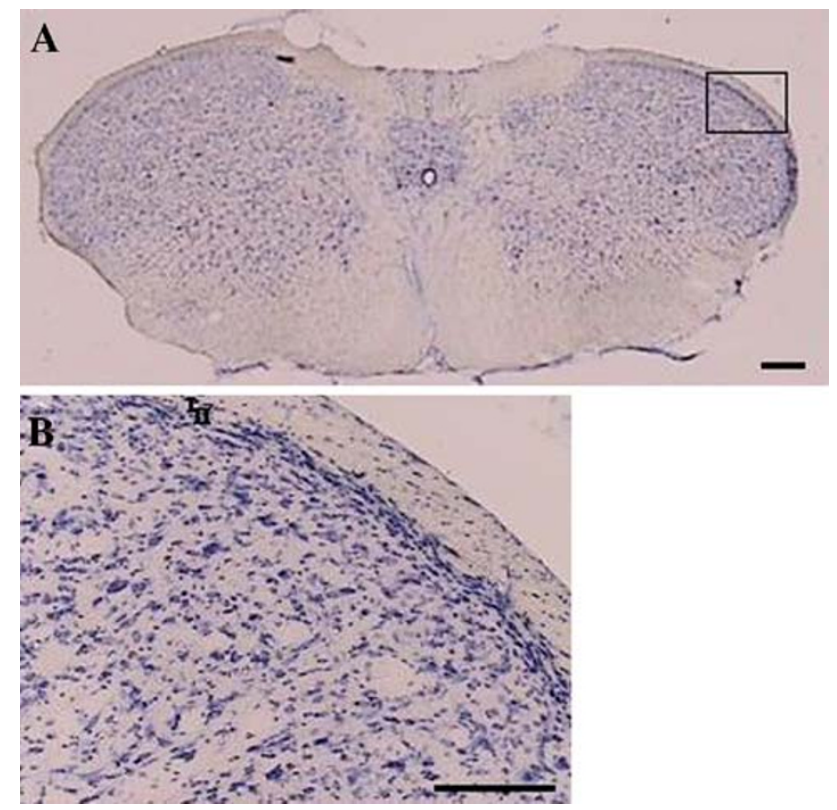

Fig. 1 Photomicrographs of Nissl-stained coronal sections of brain stem. a Brain stem, $\times 20$, scale bar $300 \mu \mathrm{c}$. b Trigeminal nucleus caudalis (TNC), $\times 200$, scale bar $150 \mu \mathrm{c}$ 
Fig. 2 Photomicrographs of the Fos expression in the TNC/II layers. a Tissue from animal that received vehicle; $\mathbf{b}$ tissue from capsaicin-injected animal; c tissue from animal that received capsaicin and melatonin; $\mathbf{d}$ tissue from pinealectomized animal; e tissue from sham-operated animal; and f tissue from pinealectomized rat that received capsaicin injection. $\times 200$, scale bar $150 \mu \mathrm{c}$. Arrow shows positivelike staining that was counted as c-fos-positive cells
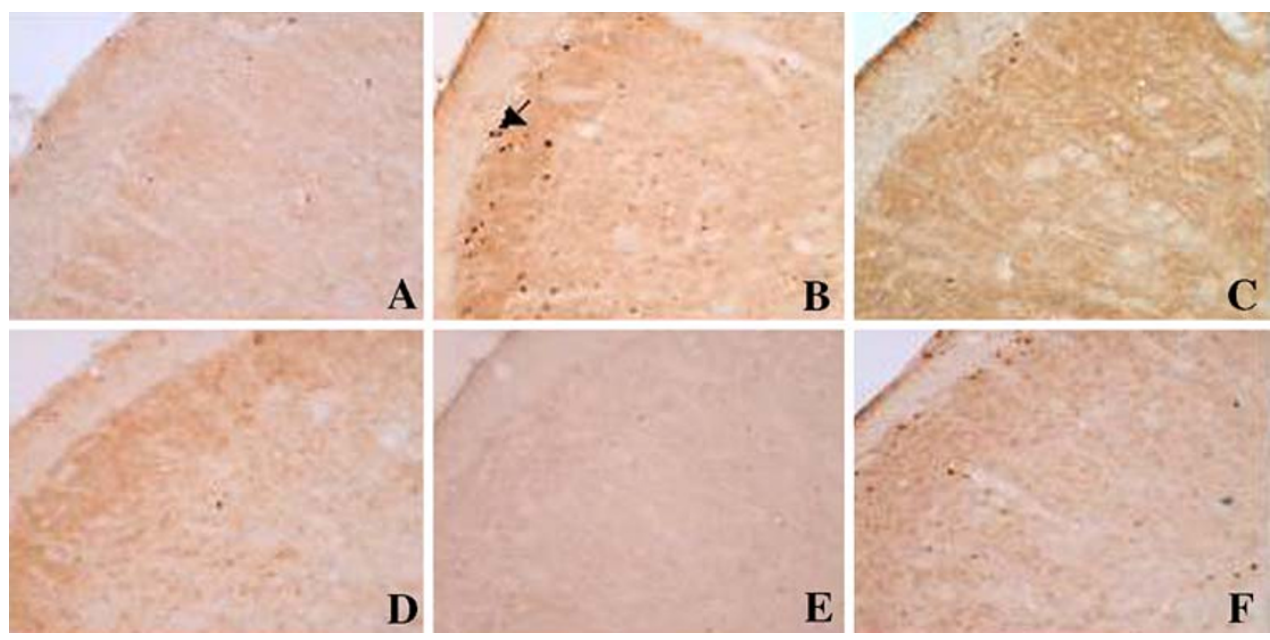

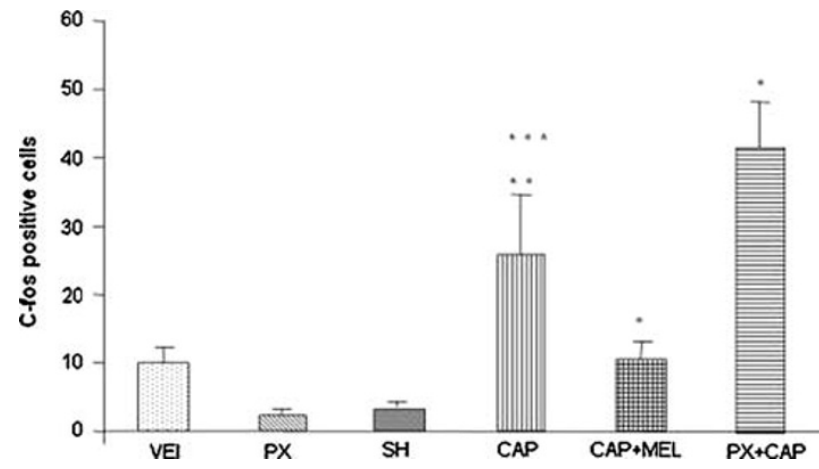

Fig. 3 Quantification of the c-fos protein immunoreactive cells in rats that received: $V E I$, vehicle $(n=5) ; S H$, sham-operated rats $(n=5) ; P X$, pinealectomized rats $(n=5) ; P X+C A P$, pinealectomized rats that received capsaicin injection $(n=7) ; C A P$, rats that received capsaicin $(n=6) ; C A P+M E L$, rats that received capsaicin and melatonin $(n=5)$ and were killed 120 min after injection. Cells were counted in $40 \mu \mathrm{m}$ sections sampled in the TNC layers I and II at 0 to $-1 \mathrm{~mm}$ caudal to obex ( 3 sections). $* P<0.01$ compared with capsaicin-treated animals; $* * P<0.001$ compared with vehicle only, sham-operated and pinealectomized rats

capsaicin-induced immunoreactive cells in TNC I and II layers in pinealectomized rats was found.

In previous studies, intracisternal capsaicin evoked c-fos expression over the rostro-caudal extent of the TNC [30, 31]. Capsaicin selectively actives nociceptive fibers and the principal nociceptive innervation of blood vessels of the subarachnoid space and dura mater originated in the trigeminal ganglion. According to Kemper [32], the number of c-fos immunoreactive cells in TNC I/II is capsaicin dose-dependent and indicates a direct and specific relationship between c-fos expression and the capsaicin concentration used. In the present work, the trigeminovascular system was activated by $200 \mathrm{nM}$ capsaicin. The ventrolateral, mediolateral and dorsomedial part of the TNC are innervated by, respectively, the ophthalmic, maxillary and mandibular branches of the trigeminal nerve.
All three branches carry fibers that innervate the meninges and meningeal vasculature [33], which were demonstrated in the present study by the increased c-fos expression in all dorsoventral parts of the TNC I/II after the intracranial capsaicin administration. This increase was intensified by the pinealectomy procedure.

Melatonin reduces lipid peroxidation process, enhances the antioxidative enzymes activities and scavenges toxicfree radical. Furthermore, melatonin inhibits the production of adhesion molecules that promote the sticking of leukocytes to endothelial cells, attenuating transendothelial cell migration and edema [34]. It inhibits the synthesis of prostaglandins acting via anti-inflammatory effect and reduces the upregulation of the pro-inflammatory cytokines, interleukins and TNF $\alpha$ [35]. Melatonin inhibits the activity of nitric oxide synthase and dopamine release, potentiates the inhibitory effect of GABA in the central nervous system, modulates the serotonin receptors and potentiates the opioid analgesic. Its structure is similar to indomethacin that inhibits COX activity [36, 37]. Thus, several mechanisms could be involved in our results.

Experimental studies demonstrated that pinealectomy increases the damage in focal cerebral ischemia and facilitates the epileptogenic process in rats, showing that melatonin has a neuroprotective action [38, 39].

In this study, we have found a great number of c-fos immunoreactive cells capsaicin induced in TNC I/II layers in pinealectomized rats, when compared with the control group, indicating that pineal gland may be potentially involved in the pathophysiology of neurovascular headaches. This finding was not observed when animals received melatonin intraperitoneally.

Several studies have evidenced low levels of melatonin in blood of patients with migraine, chronic migraine and cluster headache chronic [40, 41]. This data suggest an inadequate release of this hormone, which could be responsible for decreasing its protective role against pain, 
via several mechanisms described above. The exact mechanism, however, has yet to be determined in future studies. It is less likely that other structures or substances may play a role in these findings, since melatonin is the main secretory product of the pineal gland and no other brain structure were affected during pinealectomy.

Melatonin is a potential candidate for migraine treatment [42]. A significant headache response was found in a study looking the effect of melatonin $3 \mathrm{mg} /$ day for migraine prevention [43].

Melatonin has also been studied in cluster headache and other trigemino-autonomic cephalgias with preliminary good results. Our study is supporting melatonin supplementation as an effective suppressing agent of trigeminovascular activation in an experimental model.

\section{Conclusion}

Our data provide experimental evidence to support the role of melatonin and pineal gland in the pathophysiology of neurovascular headaches.

Conflict of interest None.

\section{References}

1. Lipton RB, Stewart WF (1998) Migraine headaches: epidemiology and comorbidity. Clin Neurosci 5:2-9

2. Russell MB (1997) Genetic epidemiology of migraine and cluster headache. Cephalalgia 17:683-701

3. Lipton RB, Stewart WF (1997) Prevalence and impact of migraine. Neurol Clin 15:1-13

4. Moskowitz MA (1984) The neurobiology of vascular head pain. Ann Neurol 16:157-168

5. Moskowitz MA (1990) Basic mechanisms in vascular headache. Neurol Clin 8:801-815

6. May A, Goadsby PJ (1999) The trigeminovascular system in humans: pathophysiologic implications for primary headache syndromes of the neural influences on the cerebral circulation. J Cereb Blood Flow Metab 19:115-127

7. Mayberg MR, Zervas NT, Moskowitz MA (1984) Trigeminal projections to supratentorial pial and dural blood vessels in cats demonstrated by horseradish peroxidase histochemistry. J Comp Neurol 223:46-56

8. Goadsby PJ, Edvinsson L (1993) The trigeminovascular system and migraine: studies characterizing cerebrovascular and neuropeptide changes seen in humans and cats. Ann Neurol 33:48-56

9. Mitsikostas DD, Sanchez del Rio M (2001) Receptor systems mediating c-fos expression within trigeminal nucleus caudalis in animal models of migraine. Brain Res Brain Res Rev 35:20-35

10. Shepheard SL, Williamson DJ, Williams J, Hill RG, Hargreaves RJ (1995) Comparison of the effects of sumatriptan and the NK1 antagonist CP-99,994 on plasma extravasation in Dura mater and c-fos mRNA expression in trigeminal nucleus caudalis of rats. Neuropharmacology 34:255-261

11. Hargreaves RJ, Shepheard SL (1999) Pathophysiology of migraine-new insights. Can J Neurol Sci 26(Suppl 3):S12-S19
12. Kaube H, Hoskin KL, Goadsby PJ (1992) Activation of the trigeminovascular system by mechanical distension of the superior sagittal sinus in the cat. Cephalalgia 12:133-136

13. Nozaki K, Boccalini P, Moskowitz MA (1992) Expression of c-fos-like immunoreactivity in brainstem after meningeal irritation by blood in the subarachnoid space. Neuroscience 49:669-680

14. Sheng M, Greenberg ME (1990) The regulation and function of c-fos and other immediate early genes in the nervous system. Neuron 4:477-485

15. Dragunow M, Robertson HA (1988) Brain injury induces c-fos protein(s) in nerve and glial-like cells in adult mammalian brain. Brain Res 455:295-299

16. Nozaki K, Boccalini P, Moskowitz MA (1992) Expression of cfos-like immunoreactivity in brainstem after meningeal irritation by blood in the subarachnoid space. Neuroscience 49:669-680

17. Kaube H, Keay KA, Hoskin KL, Bandler R, Goadsby PJ (1993) Expression of c-Fos-like immunoreactivity in the caudal medulla and upper cervical spinal cord following stimulation of the superior sagittal sinus in the cat. Brain Res 629:95-102

18. Nozaki K, Moskowitz MA, Boccalini P (1992) CP-93,129, sumatriptan, dihydroergotamine block c-fos expression within rat trigeminal nucleus caudalis caused by chemical stimulation of the meninges. Br J Pharmacol 106:409-415

19. Cutrer FM, Limmroth V, Ayata G, Moskowitz MA (1995) Attenuation by valproate of c-fos immunoreactivity in trigeminal nucleus caudalis induced by intracisternal capsaicin. Br J Pharmacol 116:3199-3204

20. Reiter RJ (1986) Normal patterns of melatonin levels in the pineal gland and body fluids of humans and experimental animals. J Neural Transm Suppl 21:35-54

21. Peres MF (2005) Melatonin, the pineal gland and their implications for headache disorders. Cephalalgia 25:403-411

22. Peres MF, Rozen TD (2001) Melatonin in the preventive treatment of chronic cluster headache. Cephalalgia 21:993-995

23. Peres MF, Masruha MR, Zukerman E, Moreira-Filho CA, Cavalheiro EA (2006) Potential therapeutic use of melatonin in migraine and other headache disorders. Expert Opin Investig Drugs 15:367-375

24. Cohen AS, Kaube H (2004) Rare nocturnal headaches. Curr Opin Neurol 17:295-299

25. Leone M, D'Amico D, Moschiano F, Fraschini F, Bussone G (1996) Melatonin versus placebo in the prophylaxis of cluster headache: a double-blind pilot study with parallel groups. Cephalalgia 16:494-496

26. Peres MF, Masruha MR, Young WB (2006) Side-shifting hemicrania continua with aura (migraine with aura with autonomic symptoms responsive to indomethacin?). Cephalalgia 26:917919

27. CIOMS (1985) CIOMS international guiding principles for biomedical research involving animals. Altern Lab Anim 12:ii

28. Siuciak JA, Dubocovich ML (1993) Effect of pinealectomy and the light/dark cycle on 2-[125I]iodomelatonin binding in the chick optic tectum. Cell Mol Neurobiol 13:193-202

29. Consiglio AR, Lucion AB (2000) Technique for collecting cerebrospinal fluid in the cisterna magna of non-anesthetized rats. Brain Res Brain Res Protoc 5:109-114

30. Cutrer FM, Limmroth V, Ayata G, Moskowitz MA (1995) Attenuation by valproate of c-fos immunoreactivity in trigeminal nucleus caudalis induced by intracisternal capsaicin. Br J Pharmacol 116:3199-3204

31. Ter Horst GJ, Meijler WJ, Korf J, Kemper RH (2001) Trigeminal nociception-induced cerebral Fos expression in the conscious rat. Cephalalgia 21:963-975

32. Kemper RH, Meijler WJ, Ter Horst GJ (1997) Trigeminovascular stimulation in conscious rats. NeuroReport 8:1123-1126 
33. Steiger HJ, Meakin CJ (1984) The meningeal representation in the trigeminal ganglion-an experimental study in the cat. Headache 24:305-309

34. Peroutka SJ (1997) Dopamine and migraine. Neurology 49:650656

35. Carrillo-Vico A, Garcia-Maurino S, Calvo JR, Guerrero JM (2003) Melatonin counteracts the inhibitory effect of PGE2 on IL-2 production in human lymphocytes via its $\mathrm{mt} 1$ membrane receptor. FASEB J 17:755-757

36. Peres MF, Masruha MR, Young WB (2006) Side-shifting hemicrania continua with aura (migraine with aura with autonomic symptoms responsive to indomethacin?). Cephalalgia 26:917919

37. Bettahi I, Pozo D, Osuna C, Reiter RJ, Acuna-Castroviejo D, Guerrero JM (1996) Melatonin reduces nitric oxide synthase activity in rat hypothalamus. J Pineal Res 20:205-210

38. Kilic E, Ozdemir YG, Bolay H, Kelestimur H, Dalkara T (1999) Pinealectomy aggravates and melatonin administration attenuates brain damage in focal ischemia. J Cereb Blood Flow Metab 19:511-516
39. de Lima E, Soares JM Jr, Carmen Sanabria GY, Gomes VS, Priel MR, Chada BE, Abrao CE, Graca Naffah-Mazzacoratti M, Amado D (2005) Effects of pinealectomy and the treatment with melatonin on the temporal lobe epilepsy in rats. Brain Res 1043:24-31

40. Brun J, Claustrat B, Saddier P, Chazot G (1995) Nocturnal melatonin excretion is decreased in patients with migraine without aura attacks associated with menses. Cephalalgia 15:136-139

41. Leone M, Lucini V, D'Amico D, Grazzi L, Moschiano F, Fraschini F, Bussone G (1998) Abnormal 24-hour urinary excretory pattern of 6-sulphatoxymelatonin in both phases of cluster headache. Cephalalgia 18:664-667

42. Miano S, Parisi P, Pelliccia A, Luchetti A, Paolino MC, Villa MP (2008) Melatonin to prevent migraine or tension-type headache in children. Neurol Sci 29:285-287

43. Peres MF, Zukerman E, da Cunha TF, Moreira FR, Cipolla-Neto $\mathrm{J}$ (2004) Melatonin, $3 \mathrm{mg}$, is effective for migraine prevention. Neurology 63:757 